\title{
HI asymmetry in the isolated galaxy CIG 85 (UGC 1547)
}

\author{
C. Sengupta ${ }^{1,2}$, T. C. Scott $^{1}$, L. Verdes Montenegro ${ }^{1}$, A. Bosma ${ }^{3}$, S. Verley ${ }^{4}$, J. M. Vilchez ${ }^{1}$, A. Durbala ${ }^{5}$, \\ M. Fernández Lorenzo ${ }^{1}$, D. Espada ${ }^{6}$, M. S. Yun ${ }^{7}$, E. Athanassoula ${ }^{3}$, J. Sulentic ${ }^{1}$, and A. Portas ${ }^{1}$ \\ ${ }^{1}$ Instituto de Astrofísica de Andalucía (IAA/CSIC), Glorieta de la Astronomia s/n, 18080 Granada, Spain \\ e-mail: sengupta@iaa.es \\ 2 Calar-Alto Observatory, Centro Astronómico Hispano Alemán, C/Jesús Durbán Remón, 2-2 04004 Almeria, Spain \\ 3 Aix Marseille Université, CNRS, LAM (Laboratoire d'Astrophysique de Marseille) UMR 7326, 13388 Marseille, France \\ 4 Dept. de Física Teórica y del Cosmos, Facultad de Ciencias, Universidad de Granada, Spain \\ 5 University of Wisconsin - Stevens Point, Department of Physics and Astronomy, 2001 Fourth Avenue, Stevens Point, \\ WI 54481-1957, USA \\ ${ }^{6}$ National Astronomical Observatory of Japan (NAOJ), 2-21-1 Osawa, Mitaka, 181-8588 Tokyo, Japan \\ 7 Department of Astronomy, University of Massachusetts, 710 North Pleasant Street, Amherst, MA 01003, USA
}

Received 4 July 2012 / Accepted 23 August 2012

\begin{abstract}
Context. We present the results from the Giant Metrewave Radio Telescope (GMRT) interferometric H I and $20 \mathrm{~cm}$ radio continuum observations of CIG 85, an isolated asymmetric galaxy from the AMIGA (Analysis of the Interstellar Medium of Isolated GAlaxies http://amiga.iaa.es) sample.

Aims. Despite being an isolated galaxy, CIG 85 showed an appreciable optical and HI spectral asymmetry and therefore was an excellent candidate for resolved HI studies to understand the reasons giving rise to asymmetries in isolated galaxies.

Methods. The galaxy was imaged in $\mathrm{H}$ I and $20 \mathrm{~cm}$ radio continuum using the GMRT. For a detailed discussion of the results we also made use of multi-wavelength data from archival SDSS, GALEX and $\mathrm{H} \alpha$ imaging.

Results. We find the H I in CIG 85 to have a clumpy, asymmetric distribution which in the NW part is correlated with optical tail like features, but the $\mathrm{H}$ I velocity field displays a relatively regular rotation pattern. Evaluating all the observational evidence, we come to a conclusion that CIG 85 is most likely a case of a disturbed spiral galaxy which now appears to have the morphology of an irregular galaxy. Although it is currently isolated from major companions, the observational evidence is consistent with $\mathrm{H}$ I asymmetries, a highly disturbed optical disk and recent increase in star formation having been caused by a minor merger, remnants of which are now projected in front of the optical disk. If this is correct, the companion will be fully accreted by CIG 85 in the near future.
\end{abstract}

Key words. galaxies: evolution - galaxies: individual: CIG 85 (UGC 1547) - radio lines: ISM - galaxies: irregular radio continuum: ISM - galaxies: spiral

\section{Introduction}

The evolution of a galaxy and its properties at $z=0$ depend on both internal processes and on its environment. To quantify the impact of different environments (nurture) on a galaxy's morphology, structure, nuclear activity, or star formation (SF) properties, amongst others, requires a well defined and statistically significant sample of minimally perturbed galaxies (pure nature). The AMIGA (Analysis of the Interstellar Medium of Isolated GAlaxies - http://amiga. iaa.es) project (Verdes-Montenegro et al. 2005) provides such a sample. The AMIGA catalogue is a refinement of the Catalogue of Isolated Galaxies (CIG; Karachentseva 1973). Galaxies in the AMIGA sample have remained free of major tidal interactions for the last 3 Gyr (Verdes-Montenegro et al. 2005). Quantification of the strength of tidal interactions with neighbouring minor companions and the local number density is available for all of the AMIGA sample galaxies (Verley et al. 2007a,b). The AMIGA project has clearly established that the most isolated galaxies have different physical properties, even compared to galaxies in what are generally considered field samples, in terms of their optical morphology (e.g. asymmetry, concentration), $L_{\mathrm{FIR}}$, radio-far infrared correlation, molecular gas content, fraction of active galactic nuclei and H I spectra asymmetry (Lisenfeld et al. 2007; Leon et al. 2008; Sabater et al. 2008; Durbala et al. 2008; Espada et al. 2011b; Lisenfeld et al. 2011).

Despite having lower rates of H I spectral and optical morphological asymmetry than galaxies in denser environments (Durbala et al. 2008; Espada et al. 2011b), some galaxies in the AMIGA sample show an appreciable asymmetry. Espada et al. (2011b) studied the H I profiles of a sample of 166 AMIGA galaxies using an $\mathrm{HI}$ asymmetry parameter $A_{\text {flux }}$, defined as the ratio of the H I flux between the receding and approaching sides of the single dish spectrum. They found the distribution of this parameter is well described by the right half of a Gaussian distribution, with only $2 \%$ of the sample having an asymmetry parameter in excess of $3 \sigma$ (i.e. $A_{\text {flux }}>1.39$ ), meaning a $39 \%$ excess of flux in one half of the spectrum. They also noted that the fraction of asymmetric H I profiles was smaller in the AMIGA sample than in any other samples in the literature. They found no correlation between the $\mathrm{HI}$ asymmetry parameter and minor companions, measured either as the tidal force (one-on-one interactions) or in terms of the number density of neighbouring galaxies. In contrast, field galaxy samples deviate from a 


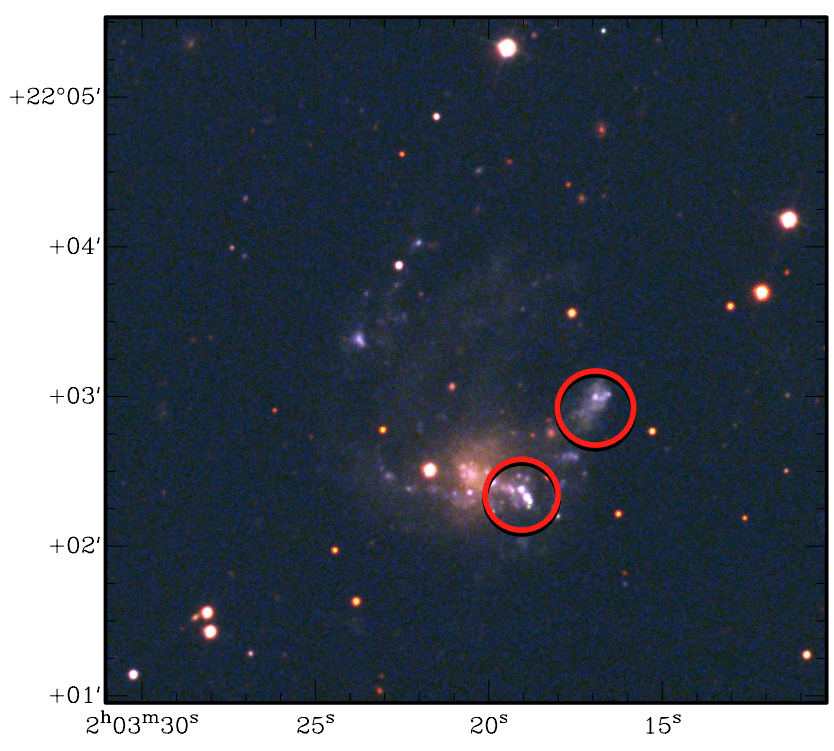

Fig. 1. CIG 85 SDSS $u, g, r$ image. The red circles indicate the positions of two possible dwarf galaxies $020036+21480-b$ (lower circle) and $020036+21480-c$ (top circle) reported by Woods et al. (2006).

Gaussian distribution and have higher (10-20\%) rates of asymmetric galaxies (Espada et al. 2011b).

It is well known that environment affects galaxy evolution: tidal interactions can stretch and deform both the stellar and gas disks, ram pressure is able to deform and strip HI disks (van Gorkom 2004), and major mergers can destroy the structure of the disks (Struck 1999). The presence of asymmetric H I profiles in isolated galaxies and the Gaussian distribution of their $A_{\text {flux }}$ parameters (Espada et al. 2011b) implies that process(es) other than major interactions and mergers are operating to maintain long lived or frequent perturbations in isolated late-type galaxies.

For isolated galaxies with H I and stellar asymmetries, several secular perturbation processes have been proposed as the cause: accretion of cold gas from the surrounding environment, intermittent accretion of satellite galaxies, and internal bar, disk or retarded SF driven perturbations together with their associated star formation (Bergvall \& Ronnback 1995; Bournaud \& Combes 2002; Bournaud et al. 2005; Sancisi et al. 2008). To date very few detailed observational studies have been carried out to determine the causes of such asymmetries in isolated galaxies.

We are therefore carrying out a programme of spatially resolved H I studies for a sample of AMIGA galaxies to determine the mechanisms that predominantly give rise to asymmetries in isolated galaxies (Espada et al. 2005, 2011b; Portas et al. 2011). As part of that study we present here Giant Metrewave Radio Telescope (GMRT) H I observations of CIG 85 (UGC 1547), (Fig. 1) which is an asymmetric AMIGA late-type galaxy with $A_{\text {flux }}=1.27 \pm 0.01$.

Basic parameters for CIG 85, which is an optically irregular galaxy, are given in Table 1. According to the AMIGA isolation criteria discussed in Verley et al. (2007b), a galaxy needs to have the density and tidal force parameters of $\eta_{\mathrm{k}} \leq 2.4$ and $Q_{\text {kar }} \leq-2.0$ respectively, to be included in the isolated sample of galaxies. These parameters for CIG 85 are 0.984 and -3.74 respectively, making it highly isolated even within the AMIGA sample. In addition we have searched the literature for companions. Arp \& Sulentic (1985) suggest this galaxy is part of a small group with three neighbours, NGC $772\left(2474 \mathrm{~km} \mathrm{~s}^{-1}\right)$, NGC $770\left(2458 \mathrm{~km} \mathrm{~s}^{-1}\right)$ and UGC $1551\left(2670 \mathrm{~km} \mathrm{~s}^{-1}\right)$. Of
Table 1. CIG 85 parameters.

\begin{tabular}{|c|c|c|}
\hline Property & Value & Reference \\
\hline$V_{\text {optical }}$ & $2640 \mathrm{~km} \mathrm{~s}^{-1}$ & Verdes-Montenegro et al. (2005) \\
\hline RA & $02^{\mathrm{h}} 03^{\mathrm{m}} 20.3^{\mathrm{s}}$ & $\begin{array}{c}\text { Leon \& Verdes- } \\
\text { Montenegro }(2003)\end{array}$ \\
\hline Dec & $+22^{\mathrm{d}} 02^{\mathrm{m}} 29.0^{\mathrm{s}}$ & $" \prime \prime \prime$ \\
\hline Distance & $35.9 \mathrm{Mpc}$ & Fernández Lorenzo et al. (2012) \\
\hline Spatial scale & $\sim 10.4 \mathrm{kpc} / \mathrm{arcmin}$ & "l \\
\hline Optical size & $2.0 \times 2.0 \operatorname{arcmin}$ & " \\
\hline Morphology & Irregular & $\prime \prime$ \\
\hline $\log \left(L_{\mathrm{B}}\right)$ & $9.47 L_{\odot}$ & " \\
\hline $\log \left(L_{\mathrm{FIR}}\right)$ & $8.86 L_{\odot}$ & AMIGA database \\
\hline
\end{tabular}

these, UGC 1551 is the nearest, projected $122^{\prime}$ (1.2 Mpc) from CIG 85. In addition to the three catalogued group members (Arp \& Sulentic 1985), a search of the NASA Extragalactic database (NED) revealed two nearer neighbours with similar optical magnitude, UGC 1538 (2835 $\left.\mathrm{km} \mathrm{s}^{-1}\right), 103^{\prime}$ (1 Mpc) away and the closest UGC 1490 (3079 $\left.\mathrm{km} \mathrm{s}^{-1}\right)$, 60' (0.6 Mpc) away. Using $200 \mathrm{~km} \mathrm{~s}^{-1}$ as the separation velocity, the time taken to cover $1 \mathrm{Mpc}$ would be $\sim 3 \mathrm{Gyr}$. This is more than twice the dynamical time scale of CIG 85. As it is generally thought that stellar and $\mathrm{H}$ I asymmetries caused by interactions dissipate within the time it takes for the galaxy to complete a single rotation, it may safely be assumed that a major interaction with known neighbours is not the cause of either the stellar or H I asymmetries observed in CIG 85. Although CIG 85 is a member of a loose group the large projected separation distances from other similar sized group members result in it having the isolation parameters of a well isolated galaxy. On the other hand, CIG 85 has two blue stellar clumps which are reported as dwarfs galaxies in Woods et al. (2006). Woods et al. (2006) suggest CIG 85 is an interacting system including the two reported dwarfs, $020036+21480-\mathrm{b}\left(02^{\mathrm{h}} 03^{\mathrm{m}} 18.8^{\mathrm{s}}+22^{\mathrm{d}} 02^{\mathrm{m}} 22.0^{\mathrm{s}} V=\right.$ $\left.2690 \mathrm{~km} \mathrm{~s}^{-1}\right)$ and $020036+21480-\mathrm{c}\left(02^{\mathrm{h}} 03^{\mathrm{m}} 16.8^{\mathrm{s}}+22^{\mathrm{d}} 03^{\mathrm{m}} 01.0^{\mathrm{s}}\right.$ $V=2618 \mathrm{~km} \mathrm{~s}^{-1}$ ) which are indicated with red circles in Fig. 1. These two objects are projected within the optical disk of CIG 85 but are not unambiguously separate galaxies.

The $L_{\text {FIR }}$ derived from the IRAS $60 \mu \mathrm{m}$ and $100 \mu \mathrm{m}$ fluxes falls within the normal range for an isolated galaxy, see Fig. 8 of Lisenfeld et al. (2007). CIG 85 H I spectra have previously been obtained from the Westerbork Synthesis Radio Telescope (WRST) (Braun et al. 2003, 49' angular resolution) and the Greenbank $91 \mathrm{~m}$ telescope (Tifft \& Cocke 1988, 10' FWHM beam). The Tifft's spectrum is shown with the dashed line in Fig. 2 and its asymmetry suggests a lopsided distribution in the H I disk. Such a perturbed distribution of stars and $\mathrm{H} \mathrm{I}$ in an apparently isolated galaxy makes CIG 85 an interesting candidate to study in detail. Our H I mapping of the galaxy (see sections below) shows this galaxy to be even more asymmetric than could be inferred from the $A_{\text {flux }}$ asymmetry parameter. This is because the $A_{\text {flux }}$ ratio parameter misses a few cases where the shape of a real asymmetric profile does not correspond to a difference in the total areas of the approaching and receding sides (Espada et al. 2011b, see for details).

We present in this paper results from the GMRT interferometric H I and $20 \mathrm{~cm}$ radio continuum observations of CIG 85 . Sloan Digital Sky Survey, SDSS (York et al. 2000), and GALEX publicly available images have also been used in this paper. Section 2 sets out details of our observations with the results given in Sect. 3 and we discuss the possible reasons for the 


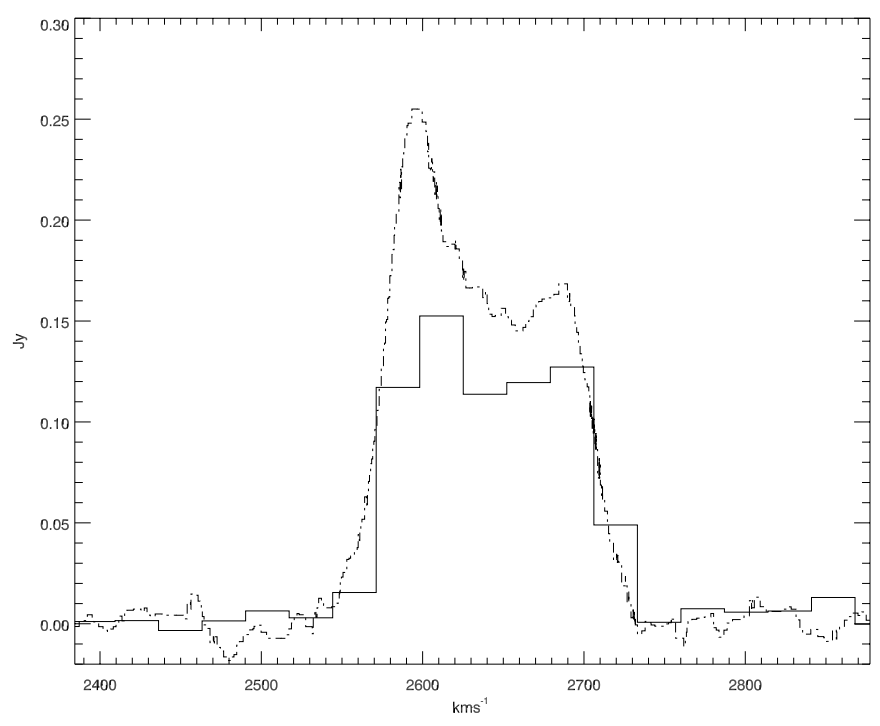

Fig. 2. H I spectra for CIG 85: Single dish from Tifft \& Cocke (1988) (dashed line) and from GMRT (solid line).

Table 2. GMRT observation details.

\begin{tabular}{lcc}
\hline \hline & H I $21 \mathrm{~cm}$ line & Radio continuum \\
\hline Observation date & 13 November 2009 & $(1405.2026 \mathrm{MHz})$ \\
Phase calibrator & $0237+288$ & $0237+288$ \\
Phase calibrator & & \\
flux density & $2.4 \mathrm{Jy} \mathrm{km} \mathrm{s}$ & \\
Integration time & $10 \mathrm{~h}$ & $2.4 \mathrm{Jy} \mathrm{km} \mathrm{s}^{-1}$ \\
rms (per channel) & $1.15 \mathrm{mJy} \mathrm{beam}^{-1}$ & $0.33 \mathrm{mJy} \mathrm{beam}^{-1}$ \\
Beam (major axis) & $24.3^{\prime \prime}$ & $47.2^{\prime \prime}$ \\
Beam (minor axis) & $21.0^{\prime \prime}$ & $37.1^{\prime \prime}$ \\
PA & $15.8^{\circ}$ & $-39.4^{\circ}$ \\
\hline
\end{tabular}

observed asymmetries in Sect. 4. Our conclusions are set out in Sect. 5. J2000 coordinates are used through the paper.

\section{Observations}

21-cm Hi line and continuum emission from CIG 85 was observed for $10 \mathrm{~h}$ with the GMRT in November, 2009. The full width at half maximum $(F W H M)$ of the GMRT primary beam at $1.420 \mathrm{GHz}$ is $\sim 24^{\prime}$. The baseband bandwidth used for $\mathrm{HI}$ line observations was $8 \mathrm{MHz}$ giving a velocity resolution of $\sim 13.7 \mathrm{~km} \mathrm{~s}^{-1}$ in the velocity range of $1800 \mathrm{~km} \mathrm{~s}^{-1}$ to $3500 \mathrm{~km} \mathrm{~s}^{-1}$. Observational parameters, including the rms noise and beam sizes used to produce the integrated maps are presented in Table 2.

The GMRT data were reduced using the Astronomical Image Processing System (AIPS) software package. Bad data due to malfunctioning antennas, antennas with abnormally low gain and/or radio frequency interference (RFI) were flagged. The primary flux density calibrators used in the observations were 3C 147 and 3C 48, and the phase calibrator was 0237+288 (see Table 2). The flux densities are on the scale of Baars et al. (1977), with flux density uncertainties of $\sim 5$ per cent. The radio continuum images were generated from the line free channels. The H I cubes were produced following continuum subtraction in the uv domain using the AIPS tasks UVSUB and UVLIN. The task IMAGR was then used to obtain the final cleaned H I cubes. From these cubes the integrated H I and H I velocity field maps were extracted using the AIPS task MOMNT. To analyse the H I and radio continuum structures in CIG 85 we produced image cubes and maps of different resolutions by tapering with different parameters, and retained for this paper the ones with beam sizes of $24.3^{\prime \prime} \times 21.0^{\prime \prime}$ and $47.1^{\prime \prime} \times 37.1^{\prime \prime}$. It is to be noted here that the shortest spacing between GMRT antennas is $\sim 60 \mathrm{~m}$ which in $L$ band imposes an angular size limit of $6^{\prime}$ to $7^{\prime}$ for the observed sources. We note that some RFI could not be completely removed and remains visible at low levels in the maps.

\section{Observational results}

\subsection{HI content and distribution}

Figure 3 shows the GMRT integrated HI maps $\left(\sim 25^{\prime \prime}\right.$ and $45^{\prime \prime}$ resolution) overlayed on an SDSS $r$-band image. The high resolution H I map (left panel) shows the HI disk to be highly disturbed with a clumpy distribution, with the large majority of the projected disk area having a column density $<10^{21}$ atoms cm ${ }^{-2}$. The high density $\mathrm{HI}$ (with maximum column densities of $1.6 \times 10^{21}$ atoms $\mathrm{cm}^{-2}$ ) is principally located in two clumps whose maxima are located $50^{\prime \prime}(8.6 \mathrm{kpc})$ south and $30^{\prime \prime}(5.2 \mathrm{kpc})$ west of the optical centre. The SDSS $r$-band image (Fig. 4) shows three prominent optical extensions (marked with red arrows) north of the optical centre. The H I disk, measured at a column density of $\sim 2 \times 10^{20}$ atoms $\mathrm{cm}^{-2}$ extends well beyond the optical extent of the galaxy, with the high density clump in the northern half of the disk coinciding with the easternmost of the optical extensions. The lower resolution H I map (right panel, Fig. 3) provides a complementary picture showing more obviously the large extent of the atomic gas in the northwest which continues well beyond the three optical extensions. The background image in this figure has been overexposed for clarity. However the H I in the far NW seems to be a continuation of the optical features. Deep optical observations of CIG 85 may reveal the presence of extended faint stellar structures in this north-western area. alternatively the absence of such structures could be used to argue for cold gas accretion, as was done in NGC 3367 where its asymmetric morphology and nuclear activity was attributed to cold gas accretion (Hernández-Toledo et al. 2011).

The integrated H I flux density obtained from Greenbank $91 \mathrm{~m}$ single dish observations of Tifft \& Cocke (1988) is $27.3 \mathrm{Jy} \mathrm{km} \mathrm{s}^{-1}$, implying an H I mass for the galaxy of $8.3 \times$ $10^{9} M_{\odot}$. Comparing this to the H I mass observed by the GMRT $\left(5.8 \times 10^{9} M_{\odot}\right)$ indicates the interferometer is only recovering $\sim 70 \%$ of H I flux and implies that CIG 85 contains a large mass of diffuse H I. We can identify where this flux difference comes from by looking at Fig. 2. There the H I spectrum from our GMRT observations (solid line) is compared to the single dish spectrum (dashed line) from Tifft \& Cocke (1988). The single dish spectrum displays a double horn profile, with a larger H I flux in the low velocity horn $\left(\sim 2580 \mathrm{~km} \mathrm{~s}^{-1}\right)$ compared to the high velocity horn $\left(\sim 2700 \mathrm{~km} \mathrm{~s}^{-1}\right)$. Our lower velocity resolution $\left(26 \mathrm{~km} \mathrm{~s}^{-1}\right)$ GMRT interferometric spectrum (solid line in Fig. 2), while having a similar velocity and velocity width, is missing flux across the range of detected velocities. However the loss is greatest in the low velocity horn and our H I velocity field (Fig. 5) shows the low velocity $\mathrm{HI}$ is located in the NW, where the HI disk is most disturbed. For this reason in all further calculations requiring the H I mass, we use the single dish value. Taking the major diameter (Table 1) as $\sim 2.0^{\prime}$ gives a $\log \frac{M_{\mathrm{H}_{\mathrm{I}}}}{D_{l}^{2}}$ value of 7.16 . A comparison with the H I surface densities of isolated galaxies of similar morphological type (Haynes \& Giovanelli 1984) shows that CIG 85 has normal H I 

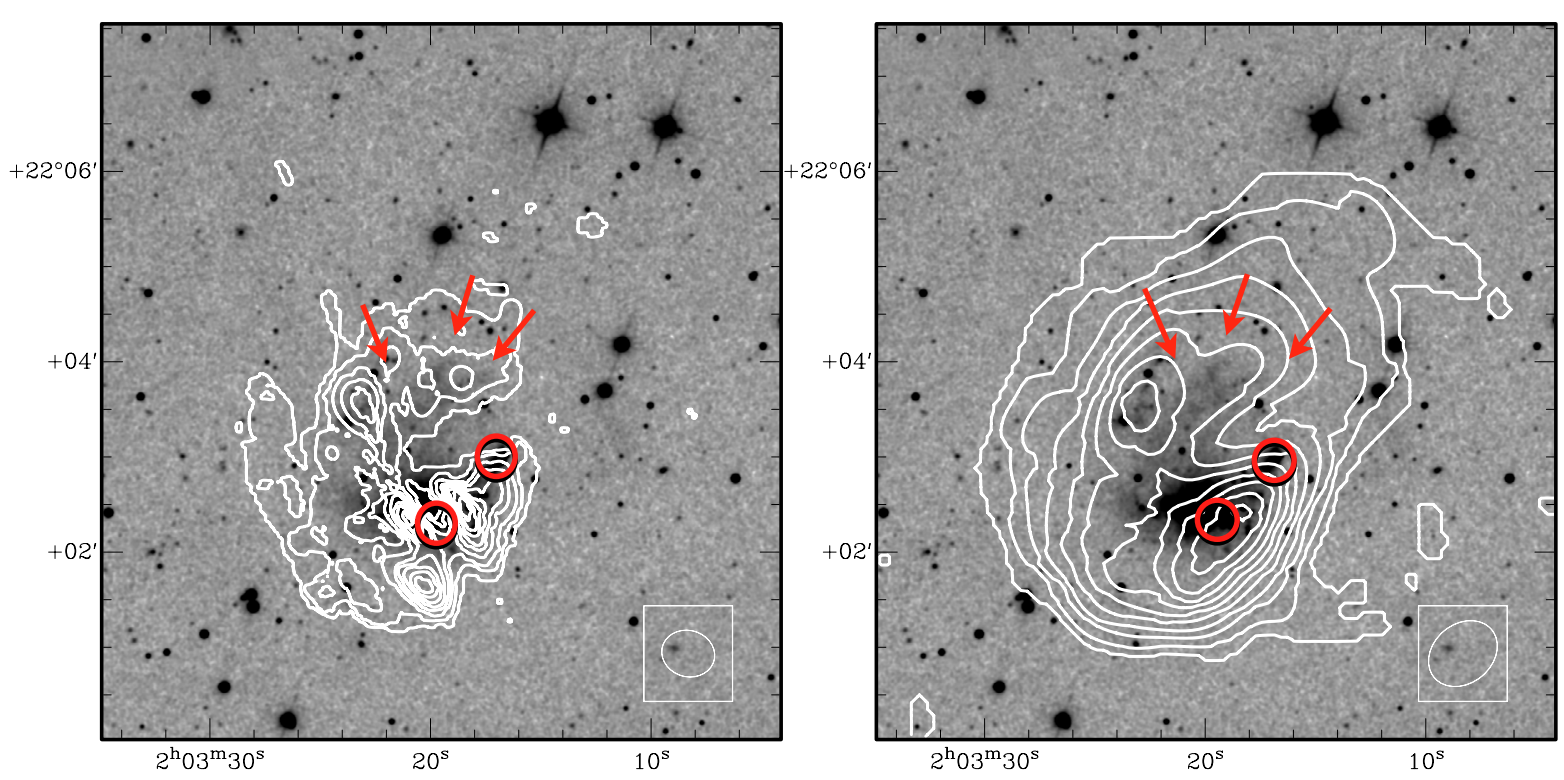

Fig. 3. Integrated HI contours on an SDSS $r$-band image, where the three faint optical arm like features are marked with red arrows and 020036+21480-b AND 020036+21480-c by circles. (Left) high resolution integrated emission, (beam size $=24.3^{\prime \prime} \times 21.0^{\prime \prime}$ ) where the H I column density levels are $10^{20}$ atoms cm$~^{-2} \times(1.0,1.9,3.2,5.3,7.5,9.6,11.8,13.9,16.1)$. (Right) low resolution integrated emission, (beam size $\left.=47.1^{\prime \prime} \times 37.1^{\prime \prime}\right)$ where the the H I column density levels are $10^{20}$ atoms $\mathrm{cm}^{-2} \times(0.56,1.2,2.2,3.4,4.7,6.0,6.9,8.2,9.4,11.0)$. The beam is shown at the bottom right of each panel.

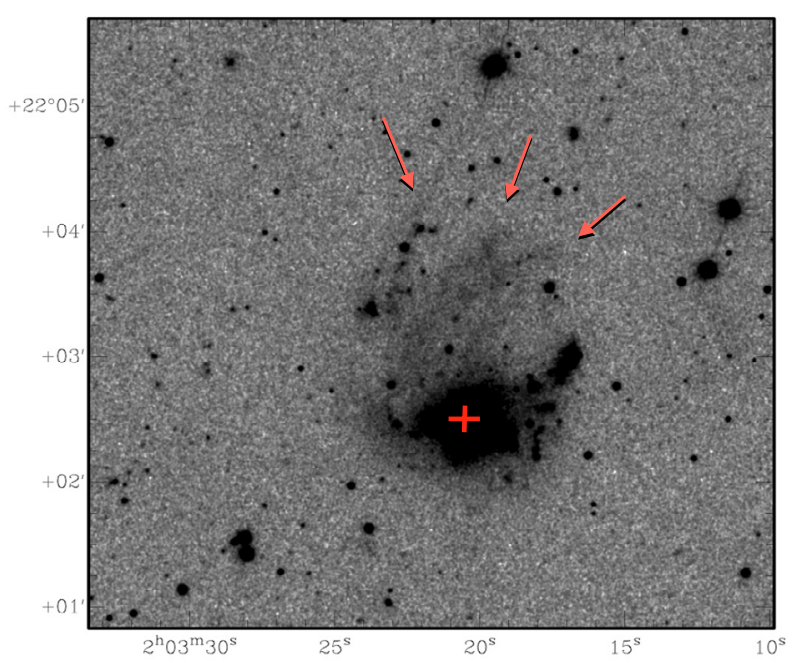

Fig. 4. SDSS $r$-band image (25-26 mag $\operatorname{arcsec}^{-2}$ ) shows faint emission to the north of the main stellar concentrations. The three faint plume or arm like features are marked with red arrows and the cross indicates the optical centre.

content. The AMIGA sample is more isolated than the Haynes \& Giovanelli (1984) sample so we compared the single dish H I mass with our AMIGA sample as well. The comparison with the AMIGA sample confirms the H I content of CIG 85 is similar to that of an isolated galaxy of its morphological type and size (Espada et al. in prep.).

The AMIGA isolation parameters are based on 2D optical data and may not include optically faint gas rich companions. Therefore, the H I cube was searched over the entire GMRT primary beam, (the $L$ band FWHM being $\sim 24^{\prime}$ which is $\sim 250 \mathrm{kpc}$ at the distance of CIG 85 ), and $\sim 1400 \mathrm{~km} \mathrm{~s}^{-1}$ velocity range, for previously undetected companions. Although the signal to noise ratio (SNR) drops considerably beyond the primary beam
FWHM, an area twice the FWHM diameter of $(\sim 0.5 \mathrm{Mpc})$ was also searched but no $\mathrm{H}$ I companions were detected in either search. The HI mass detection limit inside the FWHM of the GMRT primary beam, assuming a 3 channel line width and $3 \sigma$ SNR, is $\sim 8.5 \times 10^{7} M_{\odot}$. It is interestesting to note here that Braun et al. (2003) spectrum shows strong H I emission at velocities of $\sim 2800 \mathrm{~km} \mathrm{~s}^{-1}$ which are not present in either the Tifft $\&$ Cocke (1988) or our own GMRT spectra. The effective beam size of these observations was $\sim 49^{\prime}$. However there were baseline problems with the spectrum in this velocity range (Braun priv. comm.) and the position from which the spectrum was extracted was not stated. Therefore, though a striking feature near $2800 \mathrm{~km} \mathrm{~s}^{-1}$ is present in this spectrum, no conclusion can be drawn from it.

\subsection{HI velocity field and rotation}

In Fig. 6 we display the channel maps corresponding to the H I emission at the indicated heliocentric velocities with a channel width of $\sim 26 \mathrm{~km} \mathrm{~s}^{-1}$ and an rms noise of $0.012 \mathrm{Jy} \mathrm{beam}^{-1}$, implying a detection threshold level of $6.7 \times 10^{20}$ atoms $\mathrm{cm}^{-2}$. The H I contours have been overlayed on the $\mathrm{H}$ I integrated map displayed in grey scale. The channel maps are rather regular in velocity while clumpy in distribution, the best behaved being the channels closest to the systemic velocity. The redshifted channels show most of the HI mass is located in the eastern part, also reaching higher column densities there. In the velocity range from $2646.8 \mathrm{~km} \mathrm{~s}^{-1}$ to $2687.1 \mathrm{~km} \mathrm{~s}^{-1}$ the faintest eastern emission coincides with part of the eastern optical "plume" closer to the center of the galaxy (Fig. 4). On the other hand, the blueshifted H I is significantly more prominent in the western side where it seems to cover the outer (larger radii) parts of the three optical plumes. The velocity field, derived from the H I channel maps, is presented in Fig. 5. The main panel of Fig. 5 shows the H I velocity field derived from the high angular resolution cube, while the inset in the lower right corresponds to the 


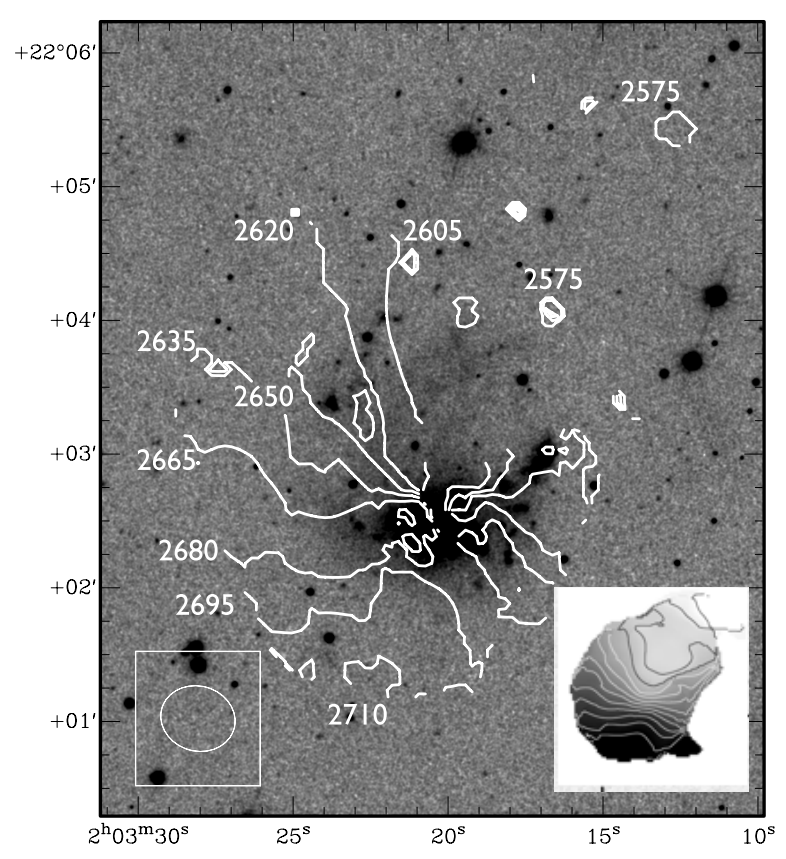

Fig. 5. H I velocity field contours derived from the high resolution cube on SDSS $r$-band band image (main panel). The velocity contours are plotted in $15 \mathrm{~km} \mathrm{~s}^{-1}$ interval. The spatial resolution is $24.3^{\prime \prime} \times 21.0^{\prime \prime}$ with the beam size indicated by the ellipse. Inset panel: low resolution $\left(47.1^{\prime \prime} \times 37.1^{\prime \prime}\right)$, systemic velocity subtracted, H I velocity field for CIG 85 . The systemic radial velocity is $2640 \mathrm{~km} \mathrm{~s}^{-1}$. From white to black, the contours (in $\mathrm{km} \mathrm{s}^{-1}$ ) are 2570, 2580, 2590, 2600, 2610, 2620, $2630,2650,2660,2670,2680,2690,2700,2710,2720$. The velocity resolution of the cube is $\sim 26 \mathrm{~km} \mathrm{~s}^{-1}$.

low resolution one, highlighting the kinematics of the more extended atomic gas. Like the channel maps, the velocity field also shows a larger regularity in the central parts at velocities closest to the systemic one. There it is consistent with a rotating disk. The northern half shows fewer isocontours due to low signal to noise $\mathrm{H}$ I detections in several places in the NW of the disk. Hints of a warped $\mathrm{HI}$ disk are seen in the velocity contours in the SE and southern edge and also in the channel maps (Fig. 6). NW of the stellar plumes the $\mathrm{HI}$ is particularly disturbed with discrete clumps of gas extending as far as $\sim 02^{\mathrm{h}} 03^{\mathrm{m}} 14.0^{\mathrm{s}}+22^{\mathrm{d}} 05^{\mathrm{m}} 30.0^{\mathrm{s}}$ with no apparent optical counterpart in the SDSS images, but maintaining a systematic gradient in velocity with radial distance $\left(2606 \mathrm{~km} \mathrm{~s}^{-1}\right.$ to $\left.2575 \mathrm{~km} \mathrm{~s}^{-1}\right)$. As noted in Sect. 3.1, most of the diffuse emission $\left(\sim 2 \times 10^{9} M_{\odot}\right)$ is expected to be located in this region. The channel maps (Fig. 6) clearly show the H I disk to be stretched in the E and NW. The kinematic centre of the galaxy is at $02^{\mathrm{h}} 03^{\mathrm{m}} 20.3^{\mathrm{s}},+22^{\mathrm{d}} 02^{\mathrm{m}} 39.8^{\mathrm{s}}$, i.e. within the uncertainties at the optical centre.

In Fig. 7 we present a position-velocity cut along a position angle of $\sim 146^{\circ}$. The direction has been selected as a compromise to include the northwestern $\mathrm{HI}$ blob, being as close to the major kinematical axis of the galaxy as possible, and avoiding the RFI effect at the galaxy centre. The zero point in the figure corresponds to $02^{\mathrm{h}} 03^{\mathrm{m}} 21.2^{\mathrm{s}}$ and $+22^{\mathrm{d}} 02^{\mathrm{m}} 47.5^{\mathrm{s}}$, while the optical centre is $02^{\mathrm{h}} 03^{\mathrm{m}} 20.3^{\mathrm{s}}+22^{\mathrm{d}} 02^{\mathrm{m}} 29.0^{\mathrm{s}}$ and the kinematic centre from the moment 1 map is estimated to be at $02^{\mathrm{h}} 03^{\mathrm{m}} 20.3^{\mathrm{s}},+22^{\mathrm{d}} 02^{\mathrm{m}} 39.8^{\mathrm{s}}$. Surprisingly, the approaching side of the position-velocity diagram is consistent with regular rotation, although this part of the galaxy appears to be more perturbed in the velocity field. The exception is the northwestern clump, which shows higher velocities than expected, suggesting it to be stripped gas. On the other hand the rotation curve in the receding side increases in a more irregular way.

From the outline of the $\mathrm{HI}$ distribution we estimate the inclination of the galaxy is $\sim 45^{\circ}$ and from the velocity width $V=$ $\frac{V_{\text {obs }}}{\sin (i)}$, we estimate the $V_{\text {rot }}=100 \mathrm{~km} \mathrm{~s}^{-1}$ implying a dynamical mass of $\sim 4.6 \times 10^{10} M_{\odot}$. Making the further assumption that the mass of molecular gas is $\simeq M(\mathrm{HI})$, the baryon fraction of CIG 85 would be $\sim 0.35$, implying a normal dark matter content similar to that found in spiral galaxies.

\section{3. $20 \mathrm{~cm}$ radio continuum from CIG 85}

$20 \mathrm{~cm}$ radio continuum was detected with a total flux density of $2.2 \mathrm{mJy}$. The lower panel of Fig. 8 shows the low resolution radio continuum emission from $\mathrm{CIG} 85$, overlayed on SDSS $r$-band image. The upper and middle panels of Fig. 8 respectively show the composite images of GALEX NUV contours with $\sim 5.3$ arcsec resolution and $\mathrm{H} \alpha$ contours from James et al. (2004) with resolution $\sim 1$ arcsec, both overlayed on SDSS $r$-band image. The radio continuum emission shows a significant overlap with the other recent SF tracers, $\mathrm{H} \alpha$ and UV (Fig. 8). The star formation rate (SFR), estimated from the $20 \mathrm{~cm}$ data, is $0.18 M_{\odot} \mathrm{yr}^{-1}$ (Yun et al. 2001) and that from the $60 \mu \mathrm{m}$ data is $\sim 0.5 M_{\odot} \mathrm{yr}^{-1}$ (Hopkins et al. 2002). Assuming that the entire radio continuum emission is non-thermal, the $\mathrm{SN}$ rate is calculated to be $0.003 \mathrm{yr}^{-1}$ (Condon \& Yin 1990). The FIR-radio relation (Yun et al. 2001) parameters for CIG 85 are, $\log L(1.4 \mathrm{GHz})=20.5$ and $\log \left(L_{60} \mu \mathrm{m}\right)=8.6$, confirming that the galaxy lies on the FIR-radio correlation (Sabater et al. 2008).

\section{Discussion}

In this section we first discuss the environment, minor companions, stellar characteristics and morphology of CIG 85, to understand the properties of this system. Then we compile all the stellar and HI observational evidence and reach a conclusion about which of the processes listed below can explain most of the observations and therefore is the likely cause of the current $\mathrm{H}$ I and stellar asymmetries of CIG 85. As an isolated galaxy, CIG 85, could be affected by any one or a combination of the following processes, which can give rise to its asymmetry.

1 Accretion of a large mass of cold gas or high velocity clouds (HVCs).

2 Internal perturbations within the disk driven by a bar.

3 Intense star formation in the disk.

4 Recent accretion of a satellite companion galaxy.

\subsection{Minor companions}

As has been mentioned in the Introduction, 020036+21480-b and $020036+21480-c$, indicated with red circles in Fig. 1, have been reported as dwarf companions of CIG 85 by Woods et al. (2006). However, the entities are projected within the disk of CIG 85, so they cannot be unambiguously categorised as separate galaxies. The AMIGA isolation parameters given in the Introduction are derived taking into account only similar sized galaxies, i.e. are designed to identify presence or absence of companions capable of producing a major interaction with the galaxy. If the tidal force parameter $Q_{\text {kar }}$ for CIG 85 is estimated taking into account all of its close neighbours, without any restriction on their size, the effect of the two dwarfs becomes significant. The value of $Q_{\mathrm{kar}}$ becomes $>0$ (well above the 

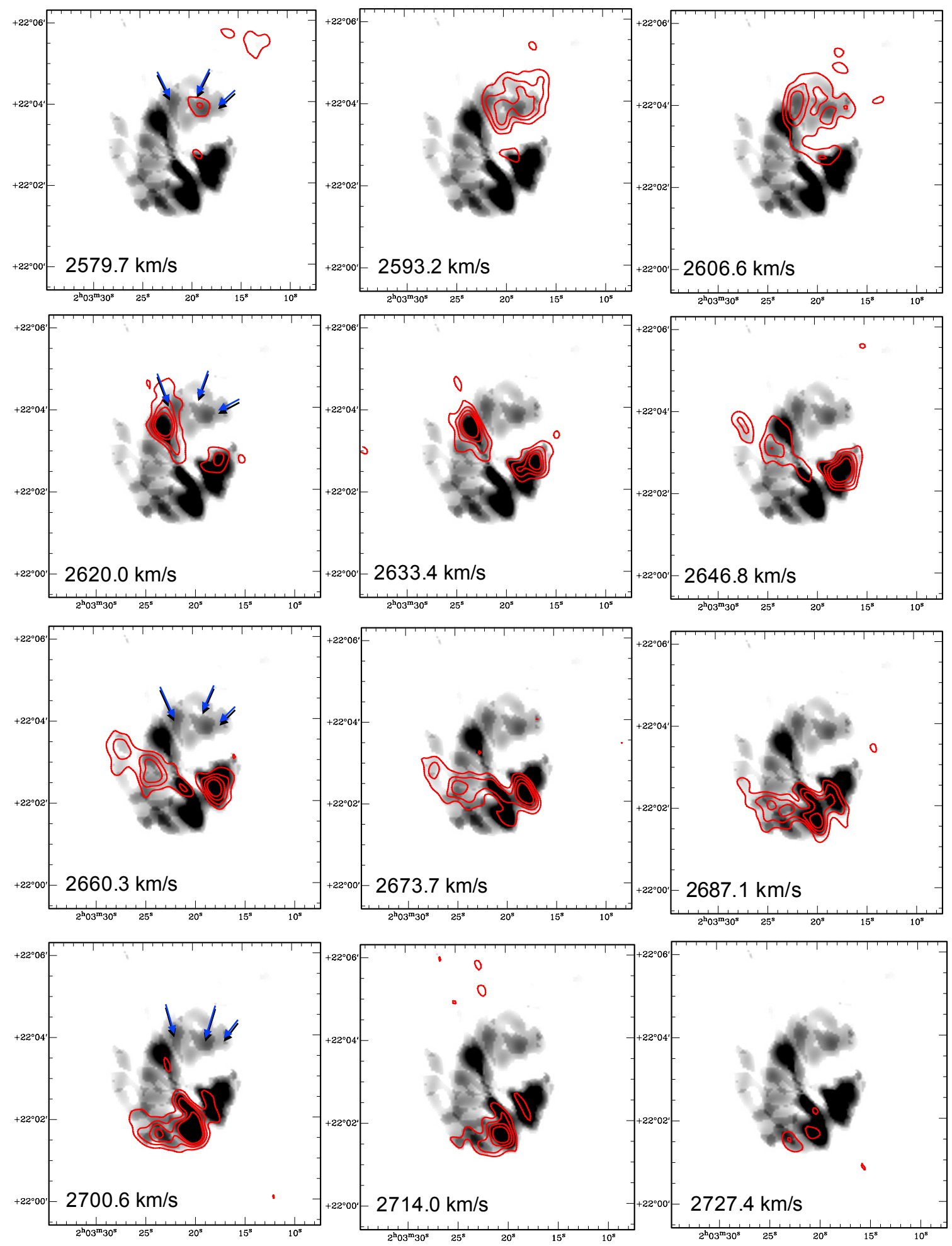

Fig. 6. Channel images (red contours) at a spatial resolution of $24.3^{\prime \prime} \times 21.0^{\prime \prime}$ overlayed on grey scale integrated HI map. The velocity in km s ${ }^{-1}$ is shown in the bottom left corner of each frame. Contour levels are at 3, 5, 7 and $9 \sigma$ where $\sigma$ corresponds to 1.4 K. Leftmost boxes of each row have three blue arrows on the galaxy to point the positions of the optical arm like features.

AMIGA cut-off value of $\leq-2.0$ ) indicating that the external tidal forces, mainly created by the very nearby companions, would be higher, and clearly dominate over the internal binding forces. So, provided the Woods et al. (2006) dwarfs are indeed entities separate from CIG 85, minor companions could be the explanation for the asymmetries observed in CIG 85.

UV (GALEX) images (Fig. 8) show the brightest SF regions within the CIG 85 disk are co-spatial with the positions of the dwarfs and the SDSS spectrum for 020036+21480-b has the characteristics of a low abundance entity. Estimated oxygen abundance from the SDSS spectrum for this object is low, about 0.2 solar abundance, typical of dwarf galaxies or the Small Magellanic Cloud. However, we only have indirect evidence suggesting the two dwarf galaxies reported by Woods et al. (2006) are individual galaxies or galaxy fragments rather than regions of high SF within CIG 85 disk, e.g. the presence of SDSS 


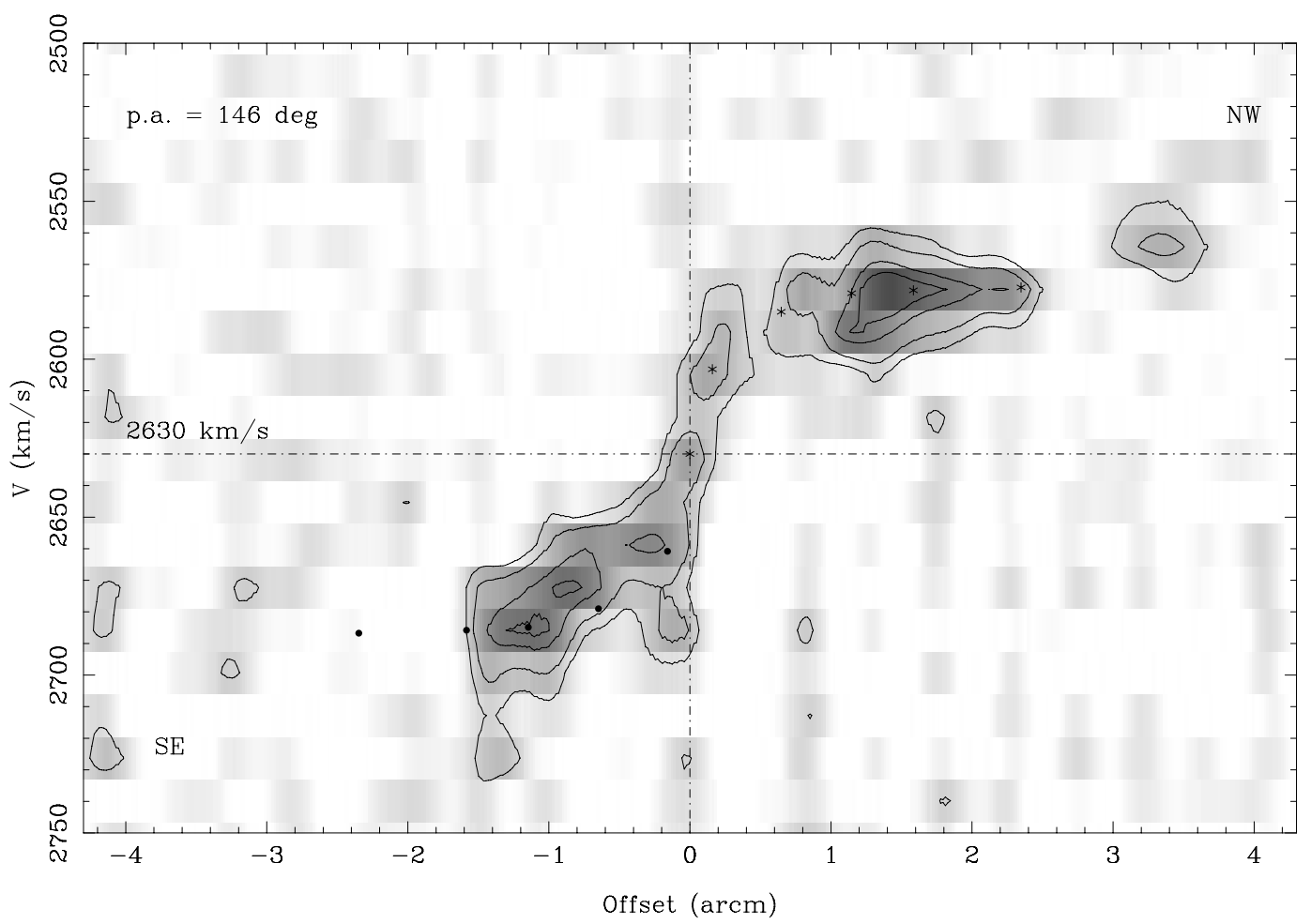

Fig. 7. Position-velocity cut along a position angle of $\sim 146^{\circ}$. The zero point in the figure corresponds to $02^{\mathrm{h}} 03^{\mathrm{m}} 21.276^{\mathrm{s}}$ and 220247.59 . The vertical line corresponds to this zero position, and the horizontal line to the velocity that maximises symmetry between the receding and approaching sides of the curve (in this particular cut, $2630 \mathrm{~km} \mathrm{~s}^{-1}$ ). The stars trace the approaching side and the dots the receding side, in order to illustrate the differences between each side.

$z$-band emission at positions of the dwarfs is consistent with separate galaxies or fragments of satellite galaxy, rather than merely young CIG $85 \mathrm{H}$ II regions. So these regions being part of the CIG 85 cannot be ruled out as well.

\subsection{Stellar properties}

The stellar mass estimated from the $L_{\mathrm{B}}$, following the method from Bell \& de Jong (2001), is $2.4 \times 10^{9} M_{\odot}$. This compares well with the stellar masses derived from the Spitzer IRAC $3.6 \mu \mathrm{m}$ and $4.5 \mu \mathrm{m}$ bands which give $1.7 \times 10^{9} M_{\odot}$ and $1.3 \times 10^{9} M_{\odot}$ respectively. Overall, the stellar mass distribution is strongly asymmetric (based on SDSS images), with most of the mass in the southern side of the galaxy, while the northern part of the disk is more diffuse (Figs. 4, 3). The SDSS $u, g, r, i$ and $z$ band images indicate a clear segregation between the distribution of the old and young stellar populations (Fig. 1). The old population is most prominent in the central region and at lower densities in faint plume-like features running north from the main concentration of stars, which could be either spiral arms or debris from an interaction (Fig. 4). In contrast the young blue stars are predominantly found in an arc which initially follows the easternmost plume (Fig. 4) and continues through the optical centre to the position of the smaller of the two dwarfs reported by Woods et al. (2006), 020036+21480-c. (Fig. 1). As expected, the UV (GALEX), H $\alpha$, radio continuum emission essentially follow the distribution of the young blue stars (Fig. 8). Both the red stellar plume and the arc of young blue stars have somewhat similar distributions to NGC 922, a collisional ring galaxy, where an off-centre high velocity collision with a companion is thought to be responsible for the $\mathrm{C}$-shaped $\mathrm{H} \alpha$ morphology and the red extended optical plumes (Wong et al. 2006). However the $\mathrm{H} \alpha$ emission in CIG 85 is dominated by the two clumps projected near the centre in contrast to NGC 922, where the emission is almost exclusively in the ring.

To place the optical morphology of CIG 85 in perspective relative to isolated AMIGA and field galaxies of similar morphological types, we estimated the asymmetry $(A)$ and concentration $(C)$ parameters in the CAS system for CIG 85. It included the "minimisation of $A$ " step required for galaxies displaying an irregular morphology, from the SDSS $r$-band image using the method from Conselice (2003). The estimated asymmetry parameter, $A=0.19 \pm 0.03$, is plotted in Fig. 9. In order to gain a perspective on the CIG $85 A$ and $C$ parameters, Fig. 9 also shows the $1 \sigma$ range of these parameters for AMIGA Sb-Sc spirals, ( $A=0.09 \pm 0.01$ Durbala et al. 2008) - red rectangle. The figure also shows the $1 \sigma$ parameter ranges for Sc-Sd spirals (solid black rectangle) and irregulars (dashed black rectangle) for the non-environmentally selected sample from Conselice (2003). While the CIG 85 CAS - $C$ parameter is very close to that of the AMIGA spirals, the $A$ parameter is clearly significantly larger than for the AMIGA spirals. The CIG 85 CAS - A parameter also falls within the upper half the Conselice $A$ parameter space for both irregular and spiral galaxies and the Conselice sample is known to include galaxies impacted by interactions.

The radial optical surface brightness profiles of a sample of late-type spirals studied by Pohlen \& Trujillo (2006) generally show an initial exponential decline associated with the bulge followed by a shallower decline in the disk. For Hubble types later than Sd the Pohlen profiles show a smaller bulge contribution leaving the profiles dominated by the more shallowly declining disk component. The surface brightness profiles for CIG 85 derived from the SDSS $g, r, i$-band images (after removal of foreground stars) all lack the bulge emission profile component typical of spirals with morphologies in the range $\mathrm{Sb}$ to Sc. However Fig. 9 shows the CAS $-C$ parameter is similar to that of AMIGA 

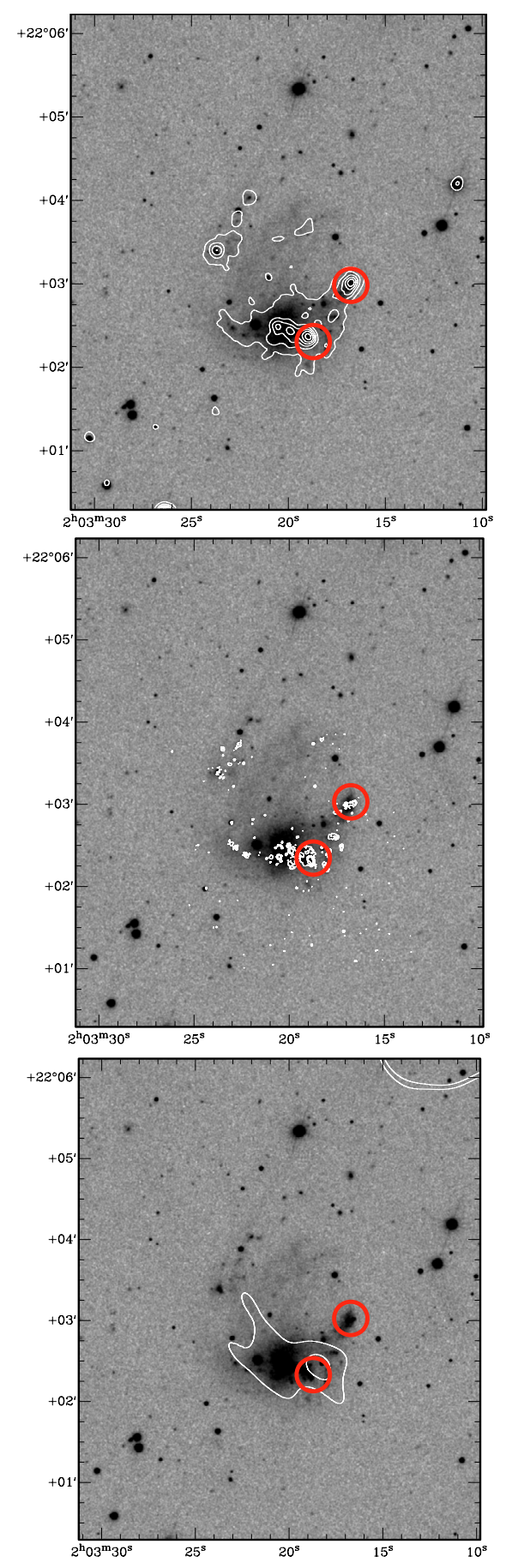

Fig. 8. Composite image of GALEX NUV contours with $\sim 5.3$ arcsec resolution (top panel), $\mathrm{H} \alpha$ contours from James et al. (2004) with resolution $\sim 1$ arcsec (middle panel) and $20 \mathrm{~cm}$ radio continuum contours with resolution $\sim 45 \operatorname{arcsec}$ (lower panel), each overlaid on an SDSS- $r$ band image. The circles indicate 020036+21480-b and 020036+21480-c.

spirals with morphologies in the range $\mathrm{Sb}$ to Sc. The most likely explanation for the apparent inconsistency between the CIG 85 CAS - $C$ parameter (consistent with a late-type spiral galaxy) and the light profile more consistent with an irregular galaxy is the presence, near the optical centre, of the two stellar clumps reported as dwarfs galaxies in Woods et al. (2006) which would artificially increase the concentration parameter.

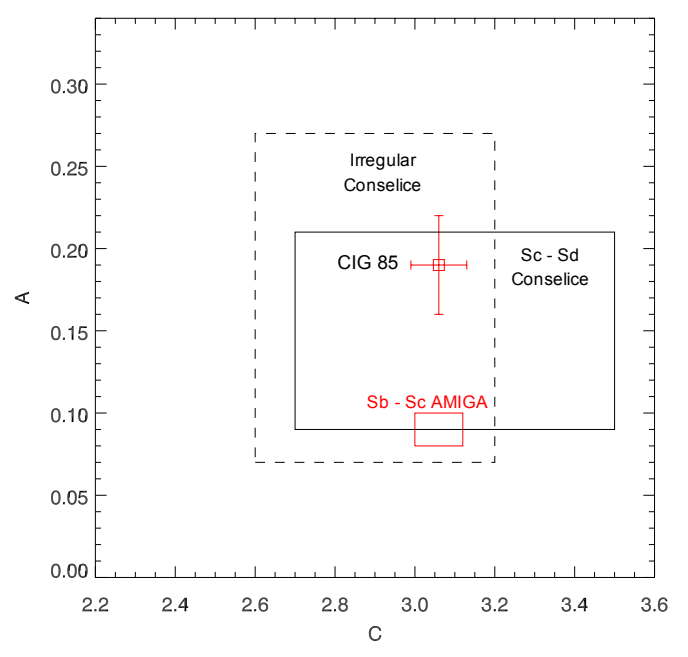

Fig. 9. CIG $85 r$-band $C$ (concentration) and $A$ (asymmetry) parameters with error bars- red point. The range of $A$ and $C$ CAS parameters for $\mathrm{Sb}-\mathrm{Sc}$ spirals (sample size $\sim 100$ ) from the AMIGA sample (Durbala et al. 2008) are indicated with the red rectangle. The range of the same $A$ and $C$ parameters from Conselice (2003) is displayed for irregular and $\mathrm{Sc}-\mathrm{Sd}$ morphologies respectively as a dashed black rectangle and a solid black rectangle.

\subsection{Morphology of CIG 85: irregular or spiral?}

Besides asymmetry, another interesting aspect of this system is its morphological type. A detailed study of its properties indicate that CIG 85 may not be a simple irregular galaxy. Several of its properties mimic a spiral, suggesting it could be a galaxy whose morphology is currently undergoing a transformation. With an optical diameter of $\sim 21 \mathrm{kpc}$ CIG 85 has a significantly greater size than the median for UGC Sm-Im irregular galaxies where $D 25=17 \mathrm{kpc}$ (Roberts \& Haynes 1994) and in general irregular galaxies are smaller in size than spirals (Pilyugin et al. 2004; Roberts \& Haynes 1994). Other properties which are more consistent with CIG 85 being an Sc-Sd spiral rather than an irregular include, the $\log \left(L_{\mathrm{B}}\right)=9.15\left[L_{\odot}\right], \log \left(L_{\mathrm{FIR}}\right)=8.75\left[L_{\odot}\right]$ (Roberts \& Haynes 1994 , UGC sample) and its CAS $-C$ parameter. The H I mass, $7.9 \times 10^{9} M_{\odot}$, of CIG 85 is also more typical of a spiral than an irregular galaxy (Roberts \& Haynes 1994). Pilyugin et al. (2004), while studying a sample of spirals and irregulars, found a clear difference between the metallicity, rotational velocity and blue band magnitudes of irregulars and spirals. Comparing CIG 85's $M_{B}$ which is -17.98 and $V_{\text {rot }} \sim 100 \mathrm{~km} \mathrm{~s}^{-1}$ to the sample of spirals and irregulars in Pilyugin et al. (2004) we find that CIG 85 has properties of a galaxy lying on, or just on the spiral side, of the irregular/spiral transition (see Figs. 9-11 of Pilyugin et al. 2004). These intermediate properties could be evidence that CIG 85 is undergoing a transformation from a spiral to an irregular or vice versa, driven by one or more of the mechanisms proposed above.

\subsection{Star formation rate and $\mathrm{HI}$ correlation}

The current global SFR estimated using the $60 \mu \mathrm{m}$ data is $\sim 0.5 M_{\odot} \mathrm{yr}^{-1}$ (Hopkins et al. 2002) and SFR $(\mathrm{H} \alpha) \sim$ $0.78 M_{\odot} \mathrm{yr}^{-1}$ (James et al. 2004). Assuming the galaxy has been forming stars over $13 \mathrm{Gyr}$, the mean $S F R$ would be $0.13 M_{\odot} \mathrm{yr}^{-1}$ indicating the current SFR is above the long term mean level. This and the fact that the regions of strongest recent SF(UV) are co-spatial with the location of the dwarfs reported by Woods et al. (2006), support the interaction induced star formation 
scenario. As was mentioned in Sect. 4.2, the stellar mass of CIG 85 is even lower than its HI mass. Also the major SF sites are close to the optical and H I kinematic centre of the galaxy and are hardly found in other parts of the disk, except for the eastern optical plume marked with an arrow in Fig. 4. These facts seem to rule out the possibility that SF activity alone is responsible for the observed large scale HI and stellar asymmetries. The current state of star formation in CIG 85 is most probably an after-effect of the asymmetries rather than the cause of them. As was noted previously, there is no marked correlation of high $\mathrm{HI}$ column density regions with the star forming regions. There are three clumps of $\mathrm{HI}$ in the disk as seen in the high resolution map (Fig. 3, left panel) having column densities $\geq 1.0 \times 10^{21}$ atoms cm $\mathrm{cm}^{-2}$. The two main clumps with column densities significantly $>1.0 \times 10^{21}$ atoms $\mathrm{cm}^{-2}$, are located towards the south. One of them correlates well with the star forming possible dwarf galaxy $020036+21480$-b and the other clump is well towards the south, away from active star forming zones. The third clump having a maximum column density $\sim 10^{21}$ atoms cm${ }^{-2}$ is in the NW part of the disk, where a blue SF region is seen in Fig. 1, although with some shift with respect to its peak. However the area looks patchy in the SDSS images, suggesting it is suffering from dust extinction which could explain this shift.

\subsection{What could have happened to CIG 85 , leading to its current morphology}

After carefully considering all the observational results presented above, we conclude that accretion of a minor companion most readily explains the current $\mathrm{H}$ I and stellar asymmetries observed in CIG 85. Under this scenario, originally CIG 85 was a gas rich spiral with a low SFR. Over the course of time the orbit of a smaller companion has decayed to the point where it has deposited tidal debris in and near the parent galaxy (e.g. the plumes seen in the $r$-band image - Fig. 4). The interaction with the satellite has resulted in the current perturbed and lopsided $\mathrm{H} \mathrm{I}$ and optical distributions. In this scenario the arc of blue stars from the east to centre would trace shock driven star formation arising from accretion of fragments of the companion, which is in a late stage of being subsumed into CIG 85. To reach this conclusion we have considered the following questions:

- Can the mechanism explain both the systematic large scale differences between diffuse northern and denser southern distributions, in both the H I and stellar components, while simultaneously accounting for the lack of correlation between the older stellar, younger stellar and H I high density regions on smaller scales?

- Are the two brightest SF regions either dwarfs/interaction remnants or SF regions within the CIG 85 disk?

- What is the interpretation of the rotation pattern in the H I disk?

- Can the scenario explain the presence of low metallicity, but not pristine, gas in the strongest SF region?

The arguments supporting the scenario described above are summarised next:

1. The CAS - A parameter which quantifies the asymmetry, is very large compared to that of the AMIGA spirals and in the upper quartile of the CAS - A parameter space of the Conselice (2003) spiral and irregular galaxy samples, which is indicative of a strong recent perturbation.
2. The detailed comparison with Roberts \& Haynes (1994) and Pilyugin et al. (2004) suggests that CIG 85 resembles in a number of ways a gas rich spiral undergoing a morphological type transition rather than an irregular.

3. The SF history of CIG 85 suggests a modest $S F R$ of $0.13 M_{\odot} \mathrm{yr}^{-1}$ in the past compared to a higher current $S F R$ of $0.5 M_{\odot} \mathrm{yr}^{-1}$. Enhanced SF is a known consequence of interactions.

4. The extended HI to the NE shows continuity with the velocity gradient of the galaxy, as well as the eastern emission visible in the $\mathrm{HI}$ channel maps (Fig. 6) from $2646.8 \mathrm{~km} \mathrm{~s}^{-1}$ to $2673.7 \mathrm{~km} \mathrm{~s}^{-1}$ consistent with these extensions being perturbed parts of the main body of the galaxy.

5. The metalicity of the gas in the $020036+21480$-b region is $\sim 0.2$ solar, consistent with that expected in objects such as SMC or a dwarf galaxy. Given the massive Hi mass of CIG 85 compared to its optical mass and its low SFR history, it is not suprising that CIG 85 will be metal poor. Additionally, this is also consistent with the idea that $020036+21480$-b region is part of a small metal poor companion galaxy whose accretion into CIG 85 is responsible for the current asymmetry.

6. The persistence of the large scale rotation pattern in the $\mathrm{HI}$ disk and the coincidence of the projected H I kinematic and optical centres of the galaxy, indicates the mass of the companion was insufficient to destroy the overall H I rotation pattern but was sufficient to significantly perturb the H I morphology.

7. The coincidence of the brightest star forming regions with $020036+21480$-b and $020036+21480$-c reported by Woods et al. (2006) as dwarf galaxies, is both consistent with accretion of a satellite and interaction induced star formation and also provides an explanation for the morphologies of red (pre-existing small bulge) and blue (blue stellar arc) stellar components.

The $A_{\text {flux }}$ asymmetry parameter of the AMIGA galaxies for which we have carried out spatially resolved H I studies as part of the AMIGA project, i.e. CIG 85, CIG 96 (Espada et al. 2005, 2011b,a) and CIG 292 (Portas et al. 2011), are 1.27, 1.16 and 1.23 respectively. Two features common to all three galaxies are the indication that the $\mathrm{H}$ I asymmetries were generated relatively recently $\left(\sim 10^{8}\right.$ yr ago $)$ and that they were not produced by interactions with major companions. Additionally in all three cases the H I velocity fields show overall regular rotation, but with indications that $\mathrm{HI}$ disks contain a warp and the signatures of $\mathrm{H}$ I asymmetry are more apparent in the $2 \mathrm{D}$ distributions than in the velocity fields. While the balance of evidence favours accretion of a minor companion as the cause of the H I asymmetries in CIG 85 and interaction with a minor companion in the case of CIG 96, it was concluded that the most likely cause of the asymmetry in CIG 292 was an internally produced perturbation.

\section{Conclusions}

Thus evaluating all the observational evidence, we come to a conclusion that CIG 85 is most likely a case of disturbed spiral galaxy which now appears to have the morphology of an irregular galaxy. Though it is currently isolated from major companions, the observational evidence is consistent with H I asymmetries, highly disturbed optical disk and recent increase in star formation having been caused by a minor merger, remnants of which are now projected in front of the optical disk. If this is correct, the companion will be fully accreted by CIG 85 in the near future. 
Acknowledgements. We thank the staff of the GMRT who have made these observations possible. GMRT is run by the National Centre for Radio Astrophysics of the Tata Institute of Fundamental Research. We thank the reviewer for his/her useful comments and suggestions. This work has been supported by Grant AYA2008-06181-C02 and AYA2011-30491-C02-01, cofinanced by MICINN and FEDER funds, and the Junta de Andalucia (Spain) grants P08-FQM4205 and TIC-114. The NASA Extragalactic Database, NED, is operated by the Jet Propulsion Laboratory, California Institute of Technology, under contract with the National Aeronautics and Space Administration. We acknowledge the usage of the HyperLeda database (http://leda.univ-lyon1.fr). Funding for the SDSS and SDSS-II has been provided by the Alfred P. Sloan Foundation, the Participating Institutions, the National Science Foundation, the US Department of Energy, the National Aeronautics and Space Administration, the Japanese Monbukagakusho, the Max Planck Society, and the Higher Education Funding Council for England. The SDSS Web Site is http://www . sdss.org/. The SDSS is managed by the Astrophysical Research Consortium for the Participating Institutions. The Participating Institutions are the American Museum of Natural History, Astrophysical Institute Potsdam, University of Basel, University of Cambridge, Case Western Reserve University, University of Chicago, Drexel University, Fermilab, the Institute for Advanced Study, the Japan Participation Group, Johns Hopkins University, the Joint Institute for Nuclear Astrophysics, the Kavli Institute for Particle Astrophysics and Cosmology, the Korean Scientist Group, the Chinese Academy of Sciences (LAMOST), Los Alamos National Laboratory, the Max-Planck-Institute for Astronomy (MPIA), the Max-Planck-Institute for Astrophysics (MPA), New Mexico State University, Ohio State University, University of Pittsburgh, University of Portsmouth, Princeton University, the United States Naval Observatory, and the University of Washington. Based on observations made with the NASA Galaxy Evolution Explorer. GALEX is operated for NASA by the California Institute of Technology under NASA contract NAS5-98034.

\section{References}

Arp, H., \& Sulentic, J. W. 1985, ApJ, 291, 88

Baars, J. W. M., Genzel, R., Pauliny-Toth, I. I. K., \& Witzel, A. 1977, A\&A, 61, 99

Bell, E. F., \& de Jong, R. S. 2001, ApJ, 550, 212

Bergvall, N., \& Ronnback, J. 1995, MNRAS, 273, 603

Bournaud, F., \& Combes, F. 2002, A\&A, 392, 83

Bournaud, F., Combes, F., Jog, C. J., \& Puerari, I. 2005, A\&A, 438, 507
Braun, R., Thilker, D., \& Walterbos, R. A. M. 2003, A\&A, 406, 829

Condon, J. J., \& Yin, Q. F. 1990, ApJ, 357, 97

Conselice, C. J. 2003, ApJS, 147, 1

Durbala, A., Sulentic, J. W., Buta, R., \& Verdes-Montenegro, L. 2008, MNRAS, 390,881

Espada, D., Bosma, A., Verdes-Montenegro, L., et al. 2005, A\&A, 442, 455

Espada, D., Muñoz-Mateos, J. C., Gil de Paz, A., et al. 2011a, ApJ, 736, 20

Espada, D., Verdes-Montenegro, L., Huchtmeier, W. K., et al. 2011b, A\&A, 532, A117

Fernández Lorenzo, M., Sulentic, J., Verdes-Montenegro, L., et al. 2012, A\&A, 540, A47

Haynes, M. P., \& Giovanelli, R. 1984, AJ, 89, 758

Hernández-Toledo, H. M., Cano-Díaz, M., Valenzuela, O., et al. 2011, AJ, 142, 182

Hopkins, A. M., Schulte-Ladbeck, R. E., \& Drozdovsky, I. O. 2002, AJ, 124, 862

James, P. A., Shane, N. S., Beckman, J. E., et al. 2004, A\&A, 414, 23

Karachentseva, V. E. 1973, Astrofizicheskie Issledovaniia Izvestiya Spetsial'noj Astrofizicheskoj Observatorii, 8, 3

Leon, S., Verdes-Montenegro, L., Sabater, J., et al. 2008, A\&A, 485, 475

Lisenfeld, U., Verdes-Montenegro, L., Sulentic, J., et al. 2007, A\&A, 462, 507

Lisenfeld, U., Espada, D., Verdes-Montenegro, L., et al. 2011, A\&A, 534, A102

Pilyugin, L. S., Vílchez, J. M., \& Contini, T. 2004, A\&A, 425, 849

Pohlen, M., \& Trujillo, I. 2006, A\&A, 454, 759

Portas, A., Scott, T. C., Brinks, E., et al. 2011, ApJ, 739, L27

Roberts, M. S., \& Haynes, M. P. 1994, ARA\&A, 32, 115

Sabater, J., Leon, S., Verdes-Montenegro, L., et al. 2008, A\&A, 486, 73

Sancisi, R., Fraternali, F., Oosterloo, T., \& van der Hulst, T. 2008, A\&ARv, 15, 189

Struck, C. 1999, Phys. Rep., 321, 1

Tifft, W. G., \& Cocke, W. J. 1988, ApJS, 67, 1

van Gorkom, J. H. 2004, in Clusters of Galaxies: Probes of Cosmological Structure and Galaxy Evolution, eds. J. S. Mulchaey, A. Dressler, \& A. Oemler, 305

Verdes-Montenegro, L., Sulentic, J., Lisenfeld, U., et al. 2005, A\&A, 436, 443

Verley, S., Odewahn, S. C., Verdes-Montenegro, L., et al. 2007a, A\&A, 470, 505

Verley, S., Leon, S., Verdes-Montenegro, L., et al. 2007b, A\&A, 472, 121

Wong, O. I., Meurer, G. R., Bekki, K., et al. 2006, MNRAS, 370, 1607

Woods, D. F., Geller, M. J., \& Barton, E. J. 2006, AJ, 132, 197

York, D. G., Adelman, J., Anderson, Jr., J. E., et al. 2000, AJ, 120, 1579

Yun, M. S., Reddy, N. A., \& Condon, J. J. 2001, ApJ, 554, 803 